\title{
Bar pressing in the rat as a function of variable visual stimulation
}

Following an adaptation period of 10 daily half hour sessions in a dark box, rats were introduced to two conditions of stimulus variability. It was found that rate of bar pressing was greater when a flickering rather than a steady light was contingent upon a response. This result was predicted by a 'stimulus change' hypothesis, although it is not inconsistent with an explanation in terms of 'scanning'.

It is now well established that rats show a significant increase over their normal operant level of bar pressing in a Skinner box if a dim light is made contingent upon this response (Kish, 1955; Marx, Henderson, \& Roberts, 1955). A number of investigators (e.g., Kish \& Antonitis, 1955; Forgays \& Levin, 1959) have suggested that the crucial reinforcing event in this situation is the stimulus change. This notion would be compatible with studies on exploration, where, for example, it has been shown that rats prefer the arm of a maze affording the greater degree of stimulus variation (Dember, Earl, \& Paradise, 1957). Yet, despite the apparent utility of the stimulus change hypothesis, it has received little direct support, and has had difficulty in coping with findings that show light offset to be a less effective reinforcer than light onset (Barnes \& Kish, 1957; Robinson, 1959).

The present experiment was designed to test the prediction from the hypothesis that the greater the variability of the stimulation contingent upon the response, then the greater the effect on response rate. Such a test was provided by Barnes \& Baron (1961), using mice. They found that the effectiveness of a visual pattern as a reinforcer was positively related to complexity; a cross being more reinforcing than a square, which in turn was more reinforcing than a circle. It is, however, difficult to define complexity either in terms of an objective physical measure, or in terms of what is discriminable to the animal. In the present experiment we compare the effectiveness of a steady versus a flickering light. These two types of stimulation should be quite readily discriminable as far as the rat is concerned, and furthermore a flickering light, with its greater variability, would constitute a greater stimulus change. Therefore, according to the stimulus change hypothesis, rats should respond more for a flickering light than for a steady light.

Method

The experimental box was sound-insulated and measured $8-1 / 2$ in. wide, $9-1 / 2$ in. long, and 10 in. deep, with a metal bar floor and lined on three walls with aluminum. The end wall had a white Perspex screen $6-1 / 4$ in. high and the full width of the wall. Threequarters of an inch below this, and 3 in. above the floor of the box was a single bar 2 in. wide extending 1 in. into the box. Frequency and duration of bar pressing were recorded on pen and cumulative recorders. The screen could be evenly illuminated from behind, the level of fllumination being matched in each stimulus condition; the meter reading at a point in the box 3 in. above the bar and 3 in. from the screen being $1.5 \mathrm{ft} .-\mathrm{c}$. When either a flickering or steady light was contingent upon a response, it remained on for 1 sec., or for as long as the bar was depressed.

Hooded rats, aged between 90 and 100 days, were used. They were raised and maintained under normal laboratory illumination, with free access to food and water. They were each placed in the box for $1 / 2 \mathrm{hr}$. a day on consecutive days. For the first 10 days the box was completely dark and a response produced no change in stimulus conditions. A baseline was thus established, on the basis of which rats were divided into three groups matched for mean response rate. Five sessions were then run in which the light group $(N=15)$ received a steady light, the flicker group ( $=$ 15) a flickering light (at 200 flashes $/ \mathrm{min}$.), and the control group $(\mathrm{N}=6)$ no illumination contingent upon a response. For six rats from each group a further five "extinction" sessions were run with no response contingent stimulation.

\section{Results}

The results are shown in Fig. 1. For statistical analysis a one-tailed Mann-Whitney $U$ test was used. In all cases the average response rate of each group per day was taken, giving $\mathrm{n}_{1}=\mathrm{n}_{2}=5$ (sessions) for each test. On the first few days of the initial 10-day period, rats showed considerable variability in response rate; however, response rate over the last five days provided a steady baseline measure. As the groups were matched, they did not differ in response rate in this period, and the control group showed no significant increase in response rate over the next five sessions $(U=8$, $p>$.25). Both light and flicker groups showed a significant increase over the baseline rate in responding during the experimental period $(U=0, p<.001)$, the flicker group responding significantly more than the light group $(U=4, p<.05)$. The light and flicker groups responded more than the control group in the "extinction" period $\left(U_{L}=5, p=.075 ; U_{F}=3, p=.048\right)$; however, 


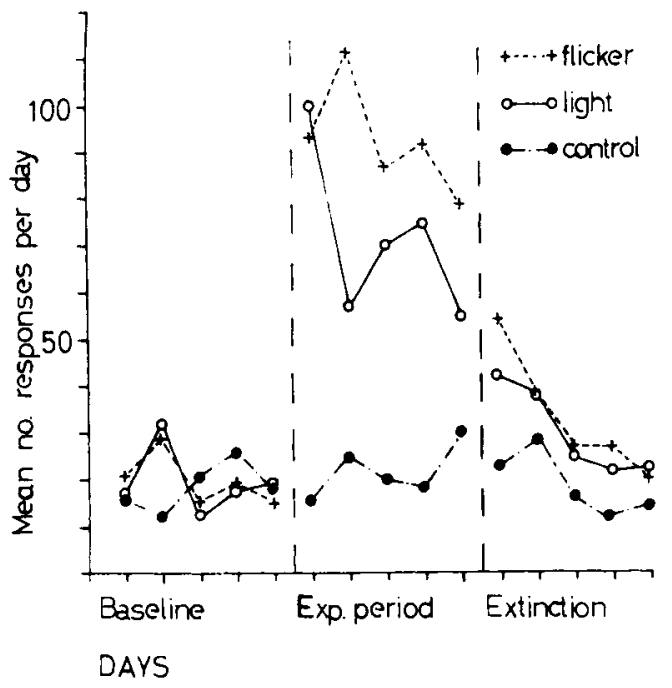

Fig. 1. Mean number of responses as a function of stimulus condition.

they themselves were not significantly different from each other.

\section{Discussion}

In accordance with the stimulus change hypothesis, the group having the flickering light should respond more frequently than the group having the steady light. This was so for the five-day experimental period. However, as can be seen from Fig. 1, this difference is not apparent on the first day on which light was introduced. A similar effect over time can be noted in the square versus cross groups in the Barnes \& Baron (1961) study, although it is there shown in a single session. The fact that the effect in the present experiment is shown only over several sessions may be due to the much longer adaptation period prior to the introduction of response contingent stimulation.

The use of a flickering light rather than a steady pattern to introduce stimulus variability presents its own difficulties, and permits an explanation of the present result in terms of the scanning hypothesis (Hurwitz, 1956; Robinson, 1959). This holds that the light provides illumination to enable the animal to scan its environment. In other words, stimulation is received both from the light itself and from the illuminated environment. A flickering light would provide less satisfactory illumination of the box than a steady light, and therefore less opportunity for scanning. While responding to the change from no light to light on the first day, the steady light group is also able to scan, familiarizing itself with both the stimulus and the interior of the box. On this first session the flicker group may not obtain as much stimulation from the environment, and so continue to respond on subsequent sessions. This hypothesis is supported by the pattern of responding of the two groups as recorded during the experimental sessions. It was found that on the first day on which light or flicker was available, both groups responded consistently throughout the session; on the second day, the flicker group continued to respond throughout the session, while most responses from the steady light group tended to be confined to the first quarter.

The finding that the experimental groups responded more than the control group in the extinction period indicates that learning had occurred in the experimental period, so providing evidence against the facilitation hypothesis (Nash \& Crowder, 1960), which states that stimulation merely increases activity. It might have been expected that the flicker group should have responded more than the light group in the extinction period, and indeed there was a slight trend in this direction; however, as the effectiveness of both reinforcers was decreasing over sessions, any differences between them may have been minimized.

The results of the experiment confirm the prediction made on the basis of stimulus change, although they are not inconsistent with an explanation in terms of scanning; these two hypotheses, however, are not necessarily mutually exclusive.

\section{References}

Bames, G. W., \& Baron, A. Stimulus complexity and sensory reinforcement. J. comp. physiol. Psychol., 1961, 54, 466-469.

Bames, G. W., \& Kish, G. B. Behavioral effects of the cessation of weak light energy. Amer. Psychologist, 1957, 12, 411.

Dember, W. N., Earl, R. W., \& Paradise, N. Response by the rat to differential stimulus complexity. J. comp. physiol. Psychol,, 1957, 50, 514-518.

Forgays, D. G., \& Levin, H. Discrimination and reversal learning as a function of change of sensory stimulation. J.comp. physiol. Psychol., 1959, 52, 191-194.

Kish, G. B. Learning when the onset of illumination is used as reinforcing stimulus. J. comp. physiol. Psychol., 1955, 48, 261-264.

Kish, G. B., \& Antonitis, J. J. Unconditioned operant behavior in two homozygous strains of mice. J. genet. Psychol., 1956, $88,121-129$.

Marx, M. H., Henderson, R. L., \& Roberts, C. L. Positive reinforcement of the bar pressing response by a light stimulus following dark operant pretests with no after-effect. J. comp. physiol. Psychol., 1955, 48, 73-76.

Nash, F. A., \& Crowder, W. F. A further study of weak-light reinforcement and response facilitation. J. Psychol., 1960, 50, 287-290.

Robinson, J. S. Light onset and termination as reinforcers for rats living under normal light conditions, Psychol. Rcp., 1959, 5, 793-796. 